\title{
Numerical Prediction of Statistical Masonry Wall Fragment Distribution Induced by Blast Loading
}

\author{
WANG Ming ${ }^{1,2}$, HAO Hong ${ }^{2}$, DING Yang ${ }^{1}$, LI Zhong-Xian ${ }^{1}$ \\ ${ }^{1}$ School of Civil Engineering, Tianjin University, Tianjin 300072, China \\ ${ }^{2}$ School of Civil and Resource Engineering, the University of Western Australia, \\ 35 Stirling Highway, Crawley WA 6009, Australia
}

\begin{abstract}
The hazard caused by the fragments of damaged structures is usually significant in accidental explosions or hostile blast events. A reliable and efficient method to estimate probable fragment size, velocity and launch distance will be very useful for engineers to assess and design counter measures to mitigate the possible fragment hazards. This paper presents a numerical method for predicting the size and launch distance of the fragments caused by explosive damage of masonry wall. Numerical simulations with different scaled distances are carried out, and the statistical distribution functions of the fragment size and launch distance in terms of the scaled distance are derived.
\end{abstract}

Keywords: Fragment size; Launch distance; masonry wall; Scaled distance; Statistical distribution function

\section{Introduction}

When an accidental explosion or a hostile blast occurs, the debris dispersion of damaged structures is one of the most significant aspects in determining the explosive hazards to nearby occupants and structures. The information about fragment size and launch distance is important for engineers to design the mitigating solutions. In the pertinent research field, majority of early effort mainly focused on the study of fracture mechanics of brittle materials ${ }^{[1-3]}$, and some of the work is based on dynamic experiments ${ }^{[4,5]}$. In the recent decades, with the development of computer techniques, many numerical simulations on fracture and fragmentation phenomenon emerge and become more popular ${ }^{[6-8]}$. However, as this research task is extremely complicated, many improvements on the existing methods are needed for reliably predicting fragments generated from damaged structures in an explosion event.

Brick masonry is one of the most common structural materials. Especially, most of historical and monumental structures, which are likely targets of terrorist attack, are made of masonry. Therefore it is important to strengthen such structures to against blast loads. In this paper, a numerical method to simulate masonry wall fragmentation is presented. A homogenized masonry wall material properties proposed by Wei and $\mathrm{Hao}^{[9]}$ is adopted to model the masonry materials. The reliability of this model as compared to the distinctive modeling is verified in a previous study. Eight numerical simulations with different scaled distances are carried out. Using the numerical results of fragment size and launch distance, statistical distribution functions of masonry wall fragments are derived in this paper. 


\section{Theory}

\subsection{Fracture mechanics and fragmentation theory}

When subjected to a tensile overstress, the preexisting mirco-cracks in brittle material would be activated and begin to grow. Certain time duration is needed for a total development of cracks. Fragment size is inextricably related with crack length. A crack growth tensor with three orthotropic components is defined as:

$$
C_{i}(t)=\int_{t_{c r}^{i}}^{t} \dot{N}_{i}(\tau) A_{i}(t-\tau) d \tau \quad i=1,2,3
$$

where $\dot{N}_{i}$ is the increase rate of the idealized penny-shaped cracks per unit area in principal direction $i$, and $A_{i}$ is the fracture region surrounding the penny-shaped flaw. $t_{c r}^{i}$ is the time duration needed for fracture to take place in direction $i$. The penny-shaped fracture region $A_{i}(t-\tau)$ formed by the growth of cracks activated at a past time $\tau$ is assumed as:

$$
A_{i}(t-\tau)=\pi c_{g}^{i 2}(t-\tau)^{2}
$$

where $c_{g}^{i}$ is the crack growth speed, which is related to the Young's modulus and density as ${ }^{[10]}$ :

$$
c_{g}^{i}=0.38 \sqrt{E_{i} / \rho}
$$

The increase rate of crack number $\dot{N}_{i}$ can be expressed as ${ }^{[2]}$ :

$$
\dot{N}_{i}=\alpha\left\langle\varepsilon_{i}-\varepsilon_{c r}^{i}\right\rangle^{\beta}
$$

$\alpha$ and $\beta$ are two material parameters, and angular bracket $\langle\cdot\rangle$ denotes a function defined as $\langle x\rangle=(|x|+x) / 2 . \quad \varepsilon_{c r}^{i}$ is the threshold tensile strain in the ith direction. In this study, an average value of strain rate is assumed in fracture derivation.

$$
\overline{\dot{\varepsilon}}_{i}=\frac{\int_{t_{c r}}^{t} \varepsilon_{i}(s) d s}{t-t_{c r}^{i}}
$$

Equation (1) can be expanded as:

$$
C_{i}(t)=\alpha \pi c_{g}^{i}{ }^{2} \dot{\dot{\varepsilon}}_{i}^{\beta} \int_{t_{c r}^{i}}^{t}\left\langle\tau-t_{c r}^{i}\right\rangle^{\beta}(t-\tau)^{2} d \tau
$$

Since cracks are assumed to grow with a constant velocity, a linear relation between crack size $r$ and time $t$ is expressed as $r=c_{g}^{i}(t-\tau)$. The crack growth tensor can then be expressed as integration of $r$

$$
\begin{gathered}
C_{i}(t)=\int_{0}^{c_{c r}^{i}\left(t-t_{c r}^{i}\right)} \omega_{i}(r, t) d r \\
\omega_{i}(r, t)=\frac{\pi \alpha r^{2}}{c_{g}^{i}}\left\langle\varepsilon_{i}(\tau)-\varepsilon_{c r}^{i}\right\rangle^{\beta}
\end{gathered}
$$

Cracks are assumed to grow in both directions simultaneously, and therefore the fragment size $S_{i}$ is twice of the crack size $r$. The fragment size distribution function $F\left(S_{i}\right)$ is

$$
F\left(S_{i}\right)=\frac{\pi \alpha S_{i}^{2}}{8 c_{g}^{i}} \dot{\bar{\varepsilon}}_{i}\left\langle t_{f}^{i}-t_{c r}^{i}-S_{i} /\left(2 c_{g}^{i}\right)\right\rangle^{\beta}
$$

When $\partial F\left(S_{i}\right) / \partial S_{i}=0$, the dominant fragment size $S_{i}^{m}$ is

$$
S_{i}^{m}=\frac{4 c_{g}^{i}}{\beta+2}\left(t_{f}^{i}-t_{c r}^{i}\right)
$$


The square root value of every component is used to denote the general fragment size.

$$
L=\sqrt{\sum_{i=1-3}\left(S_{i}^{m}\right)^{2}}
$$

\subsection{Masonry material}

In this study, the homogenized masonry material model and the corresponding material parameters presented in [9] are adopted. It includes a linear EOS, a piecewise strength criterion with a yield cap, a damage scalar, and the strain rate effect. More detailed information about the homogenized masonry model can be found in reference [9].

\section{Numerical Simulation}

The material model and fracture mechanics theory presented above are compiled as subroutines and linked with AUTODYN, and are used to calculate the fragmentation process of the example masonry wall to blast loads in this study.

\subsection{Masonry wall model and blast loads}

A masonry wall of $2.88 \mathrm{~m}$ width, $2.5 \mathrm{~m}$ height and one brick layer is modelled with homogenized masonry material properties. Every sides of the wall are assumed to be fully fixed.

Eight cases with $1000 \mathrm{~kg}$ TNT charge and different stand-off distances are simulated. Table 1 shows the blast cases.

Tab. 1 Explosive cases (1000kg TNT)

\begin{tabular}{|c|c|c|c|c|c|c|c|c|}
\hline Case No. & 1 & 2 & 3 & 4 & 5 & 6 & 7 & 8 \\
\hline Stand-off distance $(\mathrm{m})$ & 5 & 10 & 15 & 20 & 25 & 30 & 35 & 40 \\
\hline Scaled distance $\left(\mathrm{m}^{*} \mathrm{~kg}^{-1 / 3}\right)$ & 0.5 & 1.0 & 1.5 & 2.0 & 2.5 & 3.0 & 3.5 & 4.0 \\
\hline
\end{tabular}

Triangularly diminishing blast load is applied on wall surface. The peak pressure, arriving time and duration are determined by TM5- $1300^{[11]}$. In this study, explosive is assumed to be set on the ground surface, so the front surface of blast wave is semi-spherical, and blast wave arrives at the base of the wall earlier. The wall surface is divided into a number of small areas for the first three blast cases to estimate the blast load because the blast pressure in these cases is not uniform on the wall. Fig 1 shows the detail of the surface division. Because the explosive faces the center of the wall, the blast pressure is symmetric about the vertical axes, only half of the wall is analyzed. The blast pressure in each of the subdivided area is assumed uniform and calculated using a reference point at the center of the area. The blast pressures in case 4 to 8 are assumed uniform on the wall in this study because of the relatively large stand-off distance.

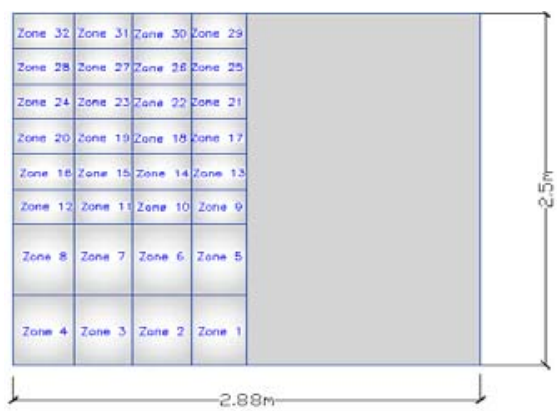

(a) Scaled distance $=0.5 \mathrm{~m}^{*} \mathrm{~kg}^{-1 / 3}$

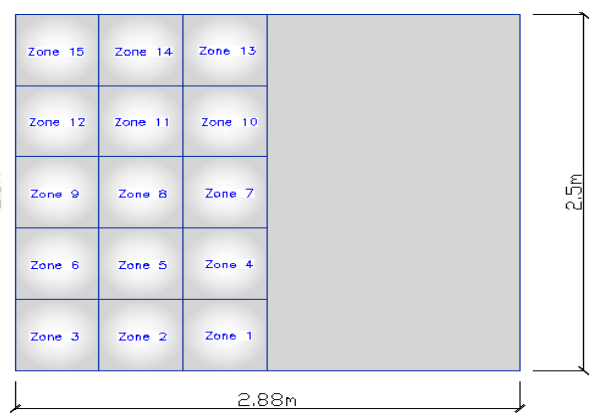

(b)Scaled distance $=1.0 \mathrm{~m}^{*} \mathrm{~kg}^{-1 / 3}$

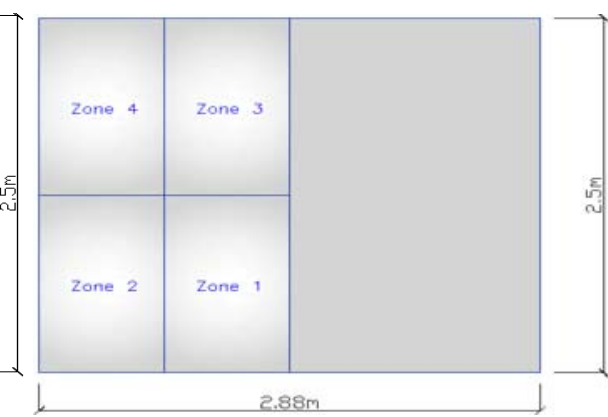

(c)Scaled distance $=1.5 \mathrm{~m} * \mathrm{~kg}^{-1 / 3}$

Fig. 1 Division of blast pressure on wall surface in the first three cases

\subsection{Fragment size distribution}

The histograms in Fig. 2 show the fragment size distributions induced in different blast cases. 


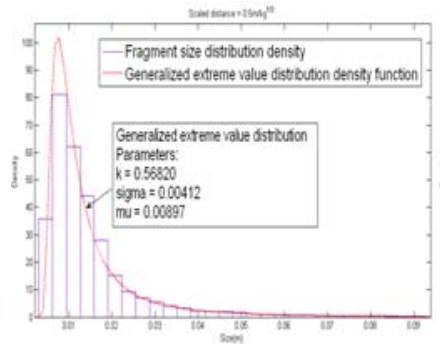

(a) Scaled distance $=0.5 \mathrm{~m}^{*} \mathrm{~kg}^{-1 / 3}$

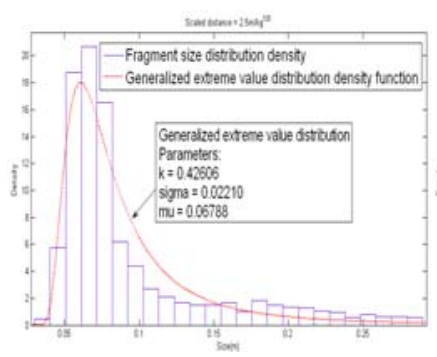

(e) Scaled distance $=2.5 \mathrm{~m}^{*} \mathrm{~kg}^{-1 / 3}$

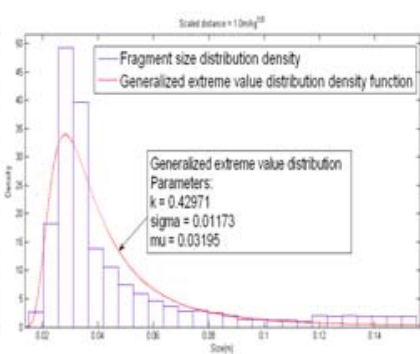

(b)Scaled distance $=1.0 \mathrm{~m}^{*} \mathrm{~kg}^{-1 / 3}$

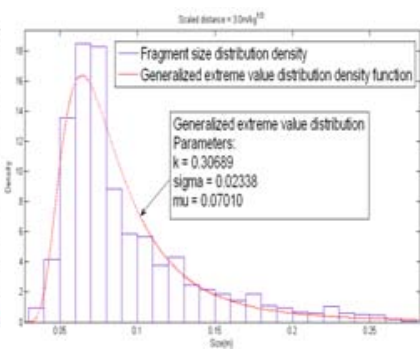

(f)Scaled distance $=3.0 \mathrm{~m}^{*} \mathrm{~kg}^{-1 / 3}$

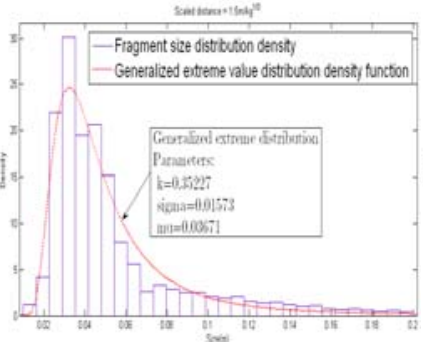

(c)Scaled distance $=1.5 \mathrm{~m}^{*} \mathrm{~kg}^{-1 / 3}$

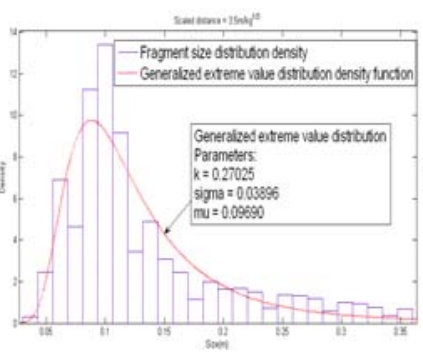

(g)Scaled distance $=3.5 \mathrm{~m}^{*} \mathrm{~kg}^{-1 / 3}$

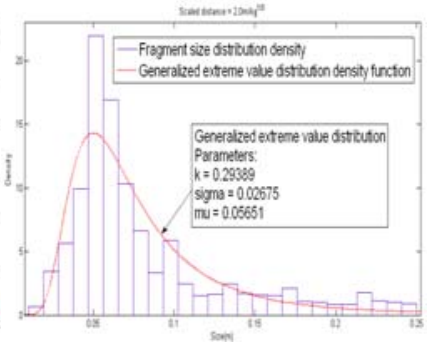

(d) Scaled distance $=2.0 \mathrm{~m} * \mathrm{~kg}^{-1 / 3}$

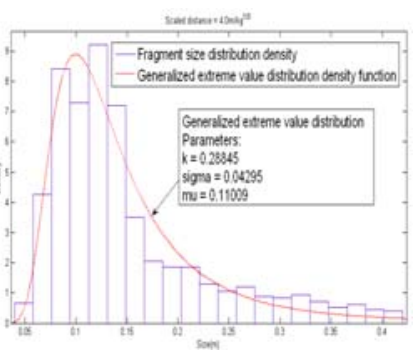

(h) Scaled distance $=4.0 \mathrm{~m} * \mathrm{~kg}^{-1 / 3}$

Fig. 2 Fragment size distribution density

According to the general distribution shapes in different cases, the generalized extreme value distribution is chosen to describe the fragment size distribution, which has a density function as:

$$
f(x ; \mu, \sigma, k)=\frac{1}{\sigma}\left[1+k\left(\frac{x-\mu}{\sigma}\right)\right]^{-1 / k-1} \exp \left\{-\left[1+k\left(\frac{x-\mu}{\sigma}\right)\right]^{-1 / k}\right\}
$$

where $\mu, \sigma, k$ are the three parameters controlling the distribution shape. The dashed lines in Fig. 2 are the generalized extreme value distribution function. As shown, it represents the fragment size distribution well.

\subsection{Fragment launch distance}

Prediction of fragment launch distance is another important aspect in evaluation of hazards caused by explosion. In this study an algorithm proposed by TM5-1300 ${ }^{[11]}$ is employed to calculate the ejection trajectory. The instantaneous velocity when cracks are totally developed is the initial condition of the launched fragment. Forces acting on the fragments and affecting their trajectories are inertia, gravitation, and fluid dynamic forces. Besides, if the formation of a fragment occurs before the explosion positive pressure elapses, blast gas pressure would also affect the trajectory.

Fluid dynamic force could be simply divided into two components called drag (along the trajectory or normal to the gravity vector) and lift (normal to the trajectory or opposing gravity) ${ }^{[11]}$.

$$
\begin{aligned}
& F_{L}=0.5 C_{L} A_{L} \rho_{a} v^{2} \\
& F_{D}=0.5 C_{D} A_{D} \rho_{a} v^{2}
\end{aligned}
$$

where $F_{L}$ and $F_{D}$ are the lift force and drag force; $C_{L}$ and $C_{D}$ are two coefficients; $A_{L}$ and $A_{D}$ are the lift area and drag area respectively. $\rho_{a}$ is the density of air, and $v$ is the fragment velocity.

In this study, fragments are assumed to be chunkily shaped, and in that case, $C_{L}$ is very small, so lift force is neglected. Taking other forces into account, we can get the following expressions of instant acceleration.

$$
\ddot{y}=\frac{2 p A-A_{D} C_{D} \rho_{a}\left(\dot{y}^{2}+\dot{z}^{2}\right) \cos \theta}{2 M}
$$




$$
\ddot{z}=-g-\frac{A_{D} C_{D} \rho_{a}\left(\dot{y}^{2}+\dot{z}^{2}\right) \sin \theta}{2 M}
$$

Where $\ddot{y}$ and $\ddot{z}$ are accelerations in horizontal and vertical directions, respectively. $p$ is the residual blast pressure at the time when fragment is formed. $\dot{y}$ and $\dot{z}$ are velocities; $g$ is the gravitational constant and $\theta$ is the trajectory angle. $A_{D}$ is the equivalent cross section area of the fragment, defined as $A_{D}=\pi L^{2} / 4$.

The rebound of fragment is not considered, and the launch distance is defined as the projected horizontal distance of the flying trajectory. Fig. 3 shows the distributions of the fragment launch distance.

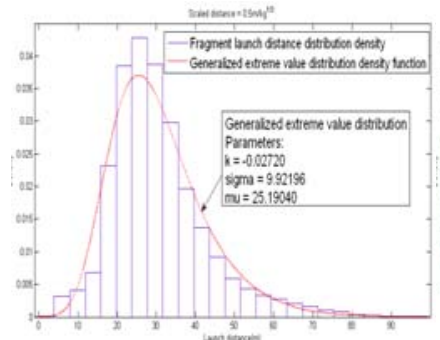

(a) Scaled distance $=0.5 \mathrm{~m} * \mathrm{~kg}^{-1 / 3}$

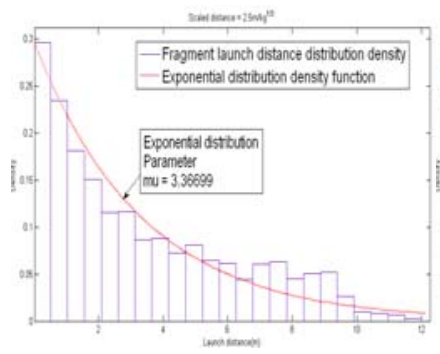

(e) Scaled distance $=2.5 \mathrm{~m}^{*} \mathrm{~kg}^{-1 / 3}$

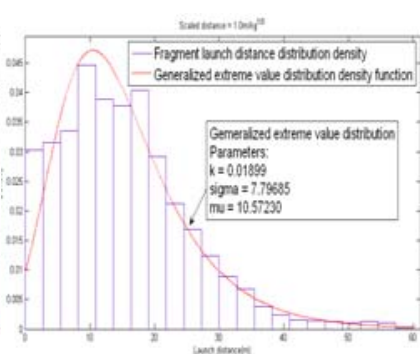

(b)Scaled distance $=1.0 \mathrm{~m}^{*} \mathrm{~kg}^{-1 / 3}$

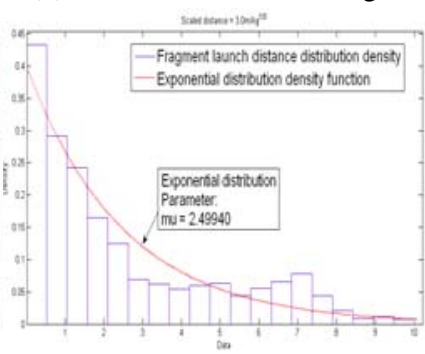

(f)Scaled distance $=3.0 \mathrm{~m}^{*} \mathrm{~kg}^{-1 / 3}$

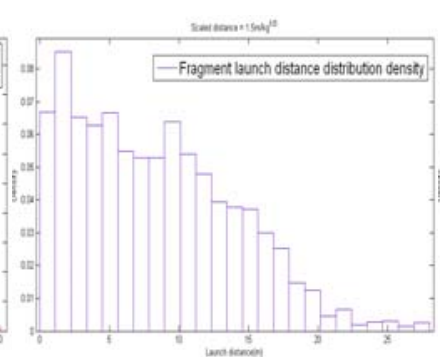

(c)Scaled distance $=1.5 \mathrm{~m}^{*} \mathrm{~kg}^{-1 / 3}$

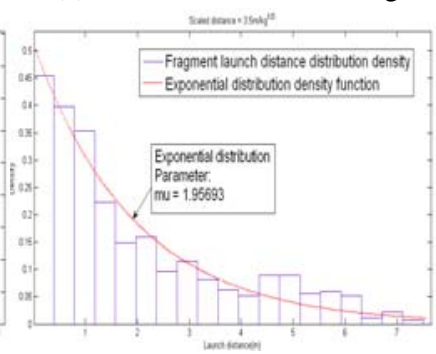

(g)Scaled distance $=3.5 \mathrm{~m}^{*} \mathrm{~kg}^{-1 / 3}$

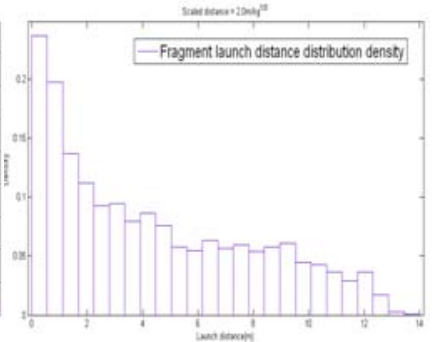

(d) Scaled distance $=2.0 \mathrm{~m} * \mathrm{~kg}^{-1 / 3}$

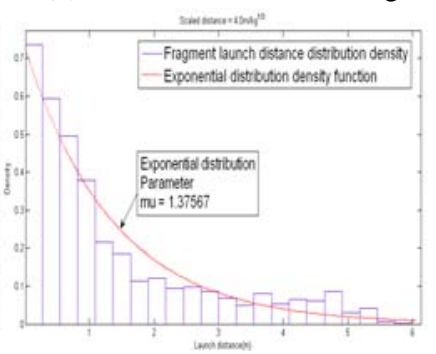

(h) Scaled distance $=4.0 \mathrm{~m}^{*} \mathrm{~kg}^{-1 / 3}$

Fig. 3 Fragment launch distance distribution density

As shown, for the first two cases, the generalized extreme value distribution is used to describe the fragment launch distance; for the last four cases, exponential distribution function is more appropriate. However, for case 3 and case 4, no suitable distribution function is found to model the distribution of fragment launch distance, which is more likely as a combination of generalized extreme value distribution and exponential distribution.

\section{Statistical properties of induced fragments}

In order to describe the relation between blast loads and fragment properties, mean and variance of fragment size and launch distance are calculated in each case, which are shown in Fig. 4 and Fig. 5.

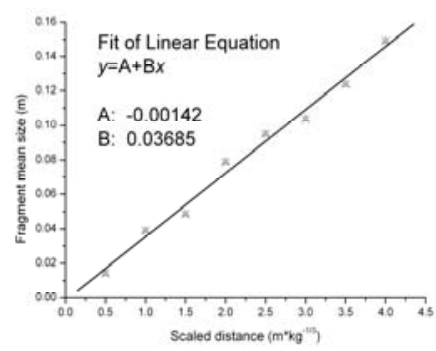

(a)Mean of fragment size

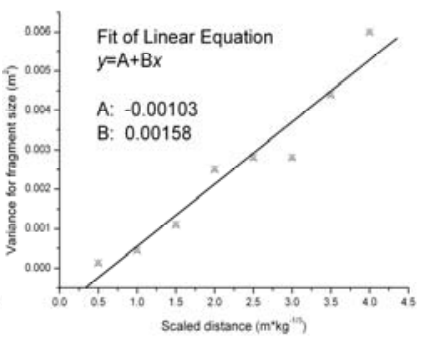

(b)Variance of fragment size

Fig. 4 Mean and variance of fragment size

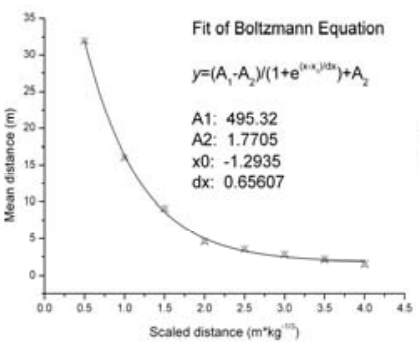

(a)Mean of launch distance

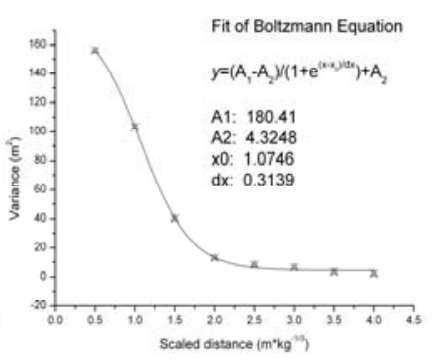

(b)Variance of launch distance

Fig. 5 Mean and variance of fragment launch distance 
According to the numerical data, linear equations are used to represent the relations between the mean fragment size, variance and the scaled distance. Similarly, the relations between mean, variance of fragment launch distance and scaled distance are described by Boltzmann Equations.

\section{Conclusions}

Numerical estimation of masonry wall fragment distributions is presented in this paper. A method based on fracture mechanics in numerical simulation is used to calculate fragment size and initial ejection velocity. The fragment launch distance is also estimated. The following conclusions can be drawn:

(1) Generalized extreme value distribution can be used to describe fragment size distribution.

(2) When the scaled distance is smaller than $1.0 \mathrm{~m} * \mathrm{~kg}^{-1 / 3}$, fragment launch distance distribution also follows the generalized extreme value distribution. When the scaled distance is bigger than $2.5 \mathrm{~m}^{*} \mathrm{~kg}^{-1 / 3}$, exponential distribution is more suitable to describe fragment launch distance distribution. When the scaled distance is between $1.0 \mathrm{~m}^{*} \mathrm{~kg}^{-1 / 3}$ and $2.5 \mathrm{~m} * \mathrm{~kg}^{-1 / 3}$, no proper distribution type for fragment launch distance is determined.

(3) Both the mean and variance of fragment size are linearly related with the scaled distance. Boltzmann Equation can be used to model the relation between mean value and variance of fragment launch distance and the scaled distance.

\section{Acknowledgements}

The authors wish to acknowledge the financial supports from the Australian Research Council (ARC) under grant number DP0774061 and the National Natural Science Foundation of China under grant number 50638030 and 50528808.

\section{References}

[1] Grady DE. Local inertial effects in dynamic fragmentation. Journal of Applied Physics 1982, 53(1):322-325.

[2] Liu LD, Katsabanis PD. Development of a continuum damage model for blasting analysis. International Journal of Rock Mechanics and Mining Science and Geomechanics Abstracts 1997, 34(2):217-231.

[3] ZHANG YQ, HAO H, LU Y. Anisotropic dynamic damage and fragmentation of rock materials under explosive loading. International Journal of Engineering Science 2003, 41: 917-929.

[4] Shockey DA, Curran DR, Seaman L, Rosenberg JT, Petersen CR. Fragmentation of rock under dynamic loads. International Journal of Rock Mechanics and Mining Sciences and Geomechanics Abstracts, 1974, 11: 303-317

[5] Grosten GA, Forsen R, Berglund R. Debris launch velocity from overloaded concrete cubicles. Proceeding of International Symposium on Interaction of the Effects of Munitions with Structures(ISIEMS), Orlando, Florida, September, 2007

[6] Camacho GT, Ortiz M. Computational modeling of impact damage in brittle materials. International Journal of Solids and Structures 1996; 33:2899-2938

[7] Kun F, Herrmann HJ. A study of fragmentation process using a discrete element method. Computer Methods in Applied Mechanics and Engineering 1996;138:3-18.

[8] Rabczuk T, Eibl J. Simulation of high velocity concrete fragmentation using SPH/MLSPH. International Journal for Numerical Methods in Engineering 2003, 56: 1421-1444

[9] Wei XY, Hao H. Numerical derivation of homogenized dynamic masonry material properties with strain rate effect. International Journal of Impact Engineering (Accepted).

[10] Kanninen MF, Popelar CH. Advanced fracture mechanics. Oxford University Press, New York 1985.

[11] TM5-1300. Structures to resist the effects of accidental explosions. US Department of the Army, Navy and Air Force Technical Manual 1990. 